\title{
A Probabilistic Environmental Decision Support Framework for Managing Risk and Resources
}

\author{
David P. Gallegos, Erik K. Webb, Paul A. Davis, and Stephen H. Conrad \\ Sandia National Laboratories \\ Albuquerque, NM, USA
}

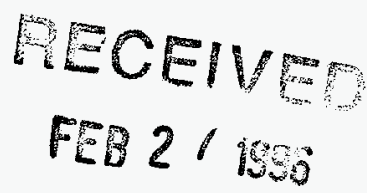

\begin{abstract}
The ability to make cost effective, timely decisions associated with waste management and environmental remediation problems has been the subject of considerable debate in recent years. On one hand, environmental decision makers do not have unlimited resources that they can apply to come to resolution on outstanding and uncertain technical issues. On the other hand, because of the possible impending consequences associated with these types of systems, avoiding making a decision is usually not an alternative either. Therefore, a structured, quantitative process is necessary that will facilitate technically defensible decision making in light of both uncertainty and resource constraints. An environmental decision support framework has been developed to provide a logical structure that defines a cost-effective, traceable, and defensible path to closure on decisions regarding compliance and resource allocation. The methodology has been applied effectively to waste disposal problems and is being adapted and implemented in subsurface environmental remediation problems.
\end{abstract}

\subsection{Introduction}

The environmental decision support framework described herein offers a generalized probabilistic framework for making consistent, defensible, and traceable environmental decisions. These decisions apply to resource management, prioritization of data collection activities, and adequacy of system performance. Most importantly, the framework provides a foundation for negotiation between owner/operators and regulators that facilitates coming to closure on decisions in a timely and cost-effective manner. Risk management, as implemented in this framework, involves the development and application of probabilistic approaches for guiding environmental restoration, risk assessment, and waste management decision making. Resource management, as guided by this framework, is accomplished through up-front, articulated objective setting, and continuous cognizance of those objectives while using sensitivity and data worth analyses to set information collection priorities. An objective of risk management in general, and this framework in particular, has been to synthesize an approach that can be commonly and collectively applied by all parties involved in environmental decisions, including regulators, site operators, policy makers, and other stakeholders.

\subsection{Existing Approaches and Structures}

Traditional approaches to evaluating risk and system performance tend to be straightforward and linear with data collection preceding the actual decision support analyses. Generally, the process begins by constructing a description of the system using existing data. This conceptualization of the system tends to describe system processes and the configuration of boundaries and internal structures with the driving purpose being simply to understand the system. This description is used as the basis for planning site characterization activities. Following this approach, because the system description can be allowed to remain vague, the characterization needs cannot help

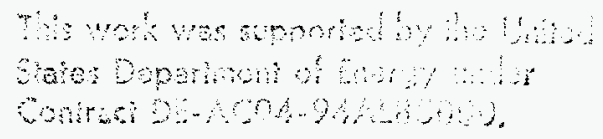




\section{DISCLAMMER}

Portions of this document may be illegible in electronic image products. Images are produced from the best available original document. 
but be somewhat ambiguous. Decision analysis, in general, involves the process of getting from the data to the decision. In the context of environmental risk management, such analyses can range from making direct inferences from the data to conducting some (typically deterministic) calculation or modeling of system behavior. If data collection has been either insufficient or superfluous, it is commonly not discovered until the end of the process. For the linear approaches, the connection between data collection and the decision to be made tends to be more implicit than explicit because the feedback loops from analysis back to data collection tend to be weak (data collection largely precedes analysis). Because traditional approaches have been recognized to be time-consuming and inefficient, improved approaches have been proposed.

The Environmental Protection Agency (EPA) has developed the Data Quality Objectives (DQOs) approach with the goal of explicitly linking data collection to the decision [1]. It formalizes the process of planning data collection by unambiguously stating the objective of the study (i.e. the decision to be made), and specifying tolerable limits on decision errors (i.e. the degree of certainty required before a decision can be made). The presumption is that reaching consensus on these issues during the planning process will allow for improved identification of the type, quantity, and quality of data that will need to be collected to support decision making. The DQO approach uses classical statistics and hypothesis testing to help make determinations on sampling design. Because the DQO approach relies on statistical inference, it is not particularly amenable to incorporating knowledge of contaminant transport processes.

The Streamlined Approach for Environmental Restoration (SAFER) combines elements of the DQO process with an approach for contingency planning to facilitate the management of uncertainty [2]. The SAFER approach provides a framework for planning and conducting remediation in a more efficient manner than more traditional approaches. It extends the DQO process by tracking implementation of the decision, looking for deviations, and having contingency plans ready. While these approaches provide improvements over more traditional, linear approaches, they are still limited by their forward-looking approach that attempts to anticipate the data required for decision making. Iterative approaches provide much stronger feedback loops, allowing for a much more explicit link from data collection to the decision.

\subsection{Description of the Decision Support Framework}

The environmental decision support framework described here utilizes an approach that incorporates process modeling, performance assessment and decision analyses, and will eventually incorporate cost/benefit analysis, and site sampling optimization techniques. Quantitative estimates of risk to human health, risk to the environment, dose to humans, or other appropriate performance measures are based on a probabilistic assessment of the site conditions. This type of analysis is desirable because it explicitly incorporates the uncertainty in information about the natural system and in turn, provides a representation of the uncertainty associated with the performance of the system; consequently, it provides a complete set of information necessary to make robust decisions regarding site safety, to direct additional site characterization, or to define site remediation schemes. The results also provide a consistent framework for comparing alternative system conceptualizations or comparing altemative engineered designs.

In developing and applying this methodology, an iterative approach has been stressed in which the definition of performance objectives sets the context of all subsequent analyses and decisions. Consequence analyses are conducted early on, and through sensitivity and data worth analyses, are used to guide the collection of site characterization data. In turn, the resulting new site characterization data are incorporated into each successive iteration of the analysis. The advantages of this approach is that decisions are focused around agreed-to performance measures, 
uncertainty in models and parameters are clearly articulated, and means are provided for tracking the reduction of the uncertainties associated with the technical analysis as more data are collected. The iterations in the process are repeated until either the uncertainty has been reduced to the point that a clear decision can be made or we find that continued data collection necessary for further reduction in uncertainty is prohibitively expensive. Ultimately, the decision makers want to minimize the probability of making a wrong decision while at the same time minimize the cost and time that it takes to make and support the decision. To address these constraints, this approach offers a logical and integrated framework for guiding technically-based decisions and for facilitating negotiations around items that are truly critical to the decisions.

\subsection{Detailed Description of Technical Components}

The decision framework is an iterative process consisting of nine steps. The integrated framework is shown in Figure 1 and the components are described in detail below.

\section{I.1 Definition of Performance Objectives}

Performance objectives are defined in the initial step in the process because they act as the driver for all subsequent steps. Defining performance measures requires an analyst to state a question or type of analysis, the alternative answers to the question, and the specific criteria that will be used to make a decision. In addition, a threshold value for the criteria (e.g., 5 ppm for a maximum concentration limit or $10^{-6}$ excess cancer risk) and probability of meeting this threshold (e.g. $95 \%$ probability of ...) must be specified. Obviously, a wide range of thresholds, metrics, and questions can be phrased in this way. Each permutation establishes a need for a slightly different analysis. Nevertheless, the generalized framework is still applicable.

\subsubsection{Data and Information Assimilation and Management}

The first iteration of the steps in the framework is based on existing data, information or knowledge. To accomplish this task the data may need to be assembled, organized and established in some form of data base. Additionally, the quality and source of information should be evaluated and maintained. Then the analyst should attempt to assimilate the existing information in order to make a site interpretation as part of the next step.

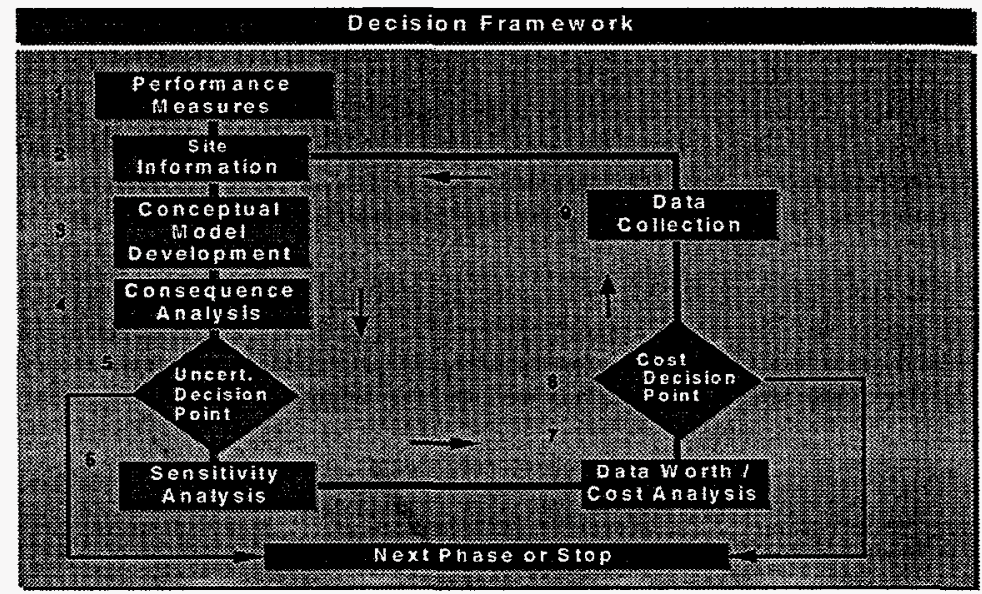

Figure 1 Environmental Decision Support Framework 


\subsubsection{Conceptual Model Development}

Conceptual model development requires an interpretation of data to develop a set of assumptions about the physical system. The uncertainty about pathways and processes (conceptual model uncertainty) as well as their controlling factors (parameter uncertainty) must also be defined. Thus, if more than one conceptual model is plausible or presented by an interested party, then each must be defined and evaluated through all steps of the decision framework to determine its validity and significance. Once these models have been developed, and documented, a probabilistic numerical simulation technique must be selected that represents the suite of specified assumptions. Then, the necessary input parameters and their associated uncertainties to perform each analysis must be defined. To do this, each parameter is defined as a distribution from which individual values are extracted for each probabilistic simulation.

\subsubsection{Consequence Analysis}

The core of this analysis is performing probabilistic physical process simulation. Generally this can be performed using Monte Carlo techniques. Then, once the simulation step is complete, the results are compiled in the form of a statistical distribution. The amount of effort and computational power necessary to accomplish this task is determined from the complexity of the conceptual model and the corresponding simulation tools.

\subsubsection{Performance Evaluation and Decision Making}

The uncertainty decision point is an evaluation of the simulation results as compared to the explicitly defined performance measures. This is generally accomplished graphically using either histograms, cumulative distribution functions, or probability maps for two and three-dimensional analysis. If the estimated distribution of concentration/risk/dose values either grossly exceeds or falls below the threshold and specified probability, an unambiguous decision can be made and defended. If the output distribution falls across the threshold and contains wide ranges of uncertainty, the user can decide to continue the analysis to define what and how much additional data collection would be of value in reducing uncertainty enough to make a decision.

\subsubsection{Sensitivity Analysis}

Sensitivity analysis is defined as a comparison of variations in input parameters with variations in the resulting output values over the previously defined distributions for each input. Techniques for performing this step include rank correlation or regression analysis on normalized input distributions. The purpose of this process is to identify those parameters which cause the greatest variance in estimates of the output based on current estimates of the input parameter distributions. The results of this analysis may help identify parameters for which collecting new data should be evaluated in the data worth step.

\subsubsection{Data Worth}

Data worth involves specifying the options one might take relative to the original question (e.g., safe or not safe, selection of remedial options) and what data one might collect to further refine the confidence in a decision. Quantitative approaches to data worth primarily involve establishing a decision tree and assigning to the component branches of the tree, probabilities of that set of conditions occurring (either from the consequence analysis or a similar computation) and the cost that would accrue if that happens. In addition, if data collection or experimental work is part of the option, the likelihood that the field or laboratory work will yield useful information is a factor in 
the probability of conditions occurring. By compiling this information, one can determine the least cost, highest probability of success, least time consuming, or some combination of the above.

\subsubsection{Cost Evaluation and Decision Making}

This step involves determining which of the various options is most cost effective. If none of the additional data collection options is cost effective, the decision maker must accept the default decision resulting from the uncertainty decision point. If one option is most cost effective, then that option (remedy, or data collection) is pursued.

\subsubsection{Data Collection}

In this step of the process, new data is collected, checked, and added to the existing data compilation. Given that the information gathered was defined through a data needs analysis, it should alter the analyst's understanding of the system and cause some change in the conceptual model, selection of numerical model, or parameter distributions. Additionally, this new data may help eliminate one or more of the alternative conceptual models. The altered conceptual model is then used in the succeeding consequence analysis and so forth.

\subsection{Application and Development of the Decision Framework}

\subsection{Low-Level Waste Disposal and Decontamination and Decommissioning}

One of the initial driving forces for the development of a software implementation of the framework (called the Sandia Environmental Decision Support System or SEDSS) was the need to provide a user-friendly platform for the Nuclear Regulatory Commission (NRC) methodology that is used in assessing the long-term performance of low-level radioactive waste (LLW) disposal sites. This user-friendly platform was also intended to supply the NRC, the states responsible for LLW disposal regulation, and site operators with a consistent technical basis for regulatory analysis of proposed sites. The LLW performance assessment methodology consists of the structured approach to decision making employed by SEDSS, models used to simulate release and transport of radionuclides from a LLW disposal facility to humans, and methods of treating model and data uncertainty. Since then, the focus of the NRC decision-related work at Sandia has been in decontamination and decommissioning (D\&D) of nuclear facilities licensed by the NRC. Here the tools developed for LLW disposal are being evaluated for their applicability to D\&D problems. Once evaluated, a new capability will be added to SEDSS to allow for the analysis of D\&D sites.

\subsection{Uranium Mill Tailings Remediation}

The Uranium Mill Tailings Remedial Action (UMTRA) program is part of the U.S. Department of Energy's effort to restore uranium mining, processing, and enriching facilities. Specifically UMTRA is responsible for 22 sites of abandoned uranium mills. As UMTRA turns its attention to groundwater remediation, there are several possible approaches that can be used for each facility. Among these is an option to perform no additional remediation and allow the natural movement of groundwater to flush contaminants to the surface drainage where they are diluted to below harmful levels. This is termed the "no-further-action" alternative. To assess whether this alternative is protective of human health, the UMTRA program is planning to apply the decision framework. This is a safety assessment with two alternative answers, "do something" or "do nothing." The determination will occur based on the sites ability to meet a specified set of concentration limits everywhere over the site at a regulatory defined 100 year compliance timeframe with a probability specified by DOE analysts. This probability has not yet been set with 
the regulatory authority, the NRC. If the "do something" alternative is chosen then a more detailed analysis, again using the framework, can be used to determine which of the proposed alternative remedies should be used and refine alternative engineering designs. Actual field implementation is planned after beta release of the SEDSS during the summer of 1996.

\subsection{Superfund}

Beginning in 1992, Sandia has provided technical assistance to EPA's Superfund program in developing improved strategies for site characterization for designing remediation and groundwater monitoring systems, and obtaining data for performing risk assessment involving the groundwater pathway. Prior to that time, the Superfund program had been criticized for mandating the implementation of ineffectual and costly site characterization strategies in their regulations and in their guidance. Implementation of these strategies led to superfluous or insufficient data collection, and inconsistent decision making. To address this problem, improved site characterization strategies for the groundwater pathway were developed, including an examination of the links between site characterization and each of the following: risk assessment, remediation selection, remedial progress tracking, and monitoring to assure contaminant containment. The synthesis of these improved strategies into a comprehensive approach resulted in the formation of the environmental decision support framework that we have presented in this paper. Since that time, the EPA Superfund program has promoted development of the SEDSS software system to automate the approaches advocated in the framework.

\subsection{Summary and Status}

The risk-based decision support framework discussed in this document will be embodied in a software tool called the Sandia Environmental Decision Support System (SEDSS). The framework and the tool are being developed to provide for more cost-effective and transparent decisions regarding environmental restoration and waste management problems. The decision framework and its embodiment in SEDSS is designed to streamline the decision making process by: (1) providing a platform for negotiation between site operators and regulators, (2) quantifying the uncertainty associated with the decision making process, and (3) extending the decision making process from the level of setting clean up criteria and acceptance levels to the level of making decisions about whether to proceed with no-further-action alternatives, to continue characterization, or to choose a remedial alternative. The methodology employed is probabilistic to provide for a direct quantitative connection between data collection and decision making, and to provide an explicit mechanism for calculating risk. This methodology relies on contaminant fate and transport modeling to allow for assessing risk level and setting clean up goals and to provide added knowledge about the extent and nature of contamination. The inclusion of cost/benefit and data optimization routines is designed to aid decision makers in choosing efficient and effective data collection and remediation strategies. Additionally, implementing the methodology into a user-friendly software system will allow for wide use and application of the methodology. And finally, the participation of the regulatory bodies, EPA and NRC, should enhance the likelihood of successful applications of the SEDSS methodology and software.

\section{References}

1. United States Environmental Protection Agency, Guidance for the data quality objectives process. EPA QA/G-4. 68 p., 1994.

2. Blacker, S.M., Cost-effective environmental restoration and corrective action using the data quality objective process. proceedings of HMC/Superfund 92, December 1-3, 1992, Washington, D.C., pp. 1022-1025, 1992. 